1. Ong CR, Molyneaux LM, Constantino M, Twigg SM, Yue DK. Longterm efficacy of metformin therapy in nonobese individuals with type 2 diabetes. Diabetes Care 2006; 29: 2361-4.

2. Solano Remírez M, González Arencibia, Álvarez Frías M, Llorente Díaz B, Echegaray Agara MN. Acidosis láctica en paciente diabético tratado con metformina. An Med Interna (Madrid) 2004; 21: 288-90.

3. Sánchez R, Manteiga Riestra E, Martínez González O. Metformin-induced acute lactic acidosis. Farm Hosp 2007; 31: 71-2

4. Orban JC, Ghaddab A, Chatti O, Ichai C. Metformin-associated lactic acidosis. Ann Fr. Anesth Reanim 2006; 25: 1046-52

5. Lalau JD, Race JM. Lactic acidosis in metformin therapy: Searching for a link with metformin in reports of metformin-associated lactic acidosis. Diabetes Obes Metab 2001: 195-201.

6. Vidal S, Mariot J, Galy-Floc'h M, Azoulay E. Metformin-associated lactic acidosis precipitated by acute renal failure. Ann Fr Anesth Rea$\operatorname{nim} 2003$; 22: 457-60.

\section{Derrame pleural asociado a diclofenaco}

\section{Sr. Director:}

Los antiinflamatorios no esteroideos (AINE) son un grupo de fármacos utilizados frecuentemente, la acción terapéutica y la toxicidad dependen en gran parte de la inhibición de la síntesis de prostaglandinas. Se presenta el caso de un paciente en tratamiento con diclofenaco que desarrolló un derrame pleural.

Varón de 37 años de edad, de raza negra, fumador de 20 cigarrillos/día, y no tenía enfermedades de interés. Un mes antes de la consulta presentó dolor en la rodilla izquierda por lo que inició tratamiento con diclofenaco $50 \mathrm{mg} / 8$ horas. A los 20 días de iniciar la terapia presentó edemas en miembros inferiores y se realizaron un análisis apareciendo hgb 7,9 g/dl, VCM 64 fl, y una radiografía de tórax en la que se observaba un derrame pleural bilateral (Fig. 1).

Presentaba una TA 195/116 mmHg, y la auscultación cardíaca reveló un soplo sistólico. La bioquímica creatinina, ácido úrico, calcio, fosfato, colesterol, proteínas totales, GTP, GGT, sodio y potasio normales. Proteinograma: aumento policlonal de gamma-

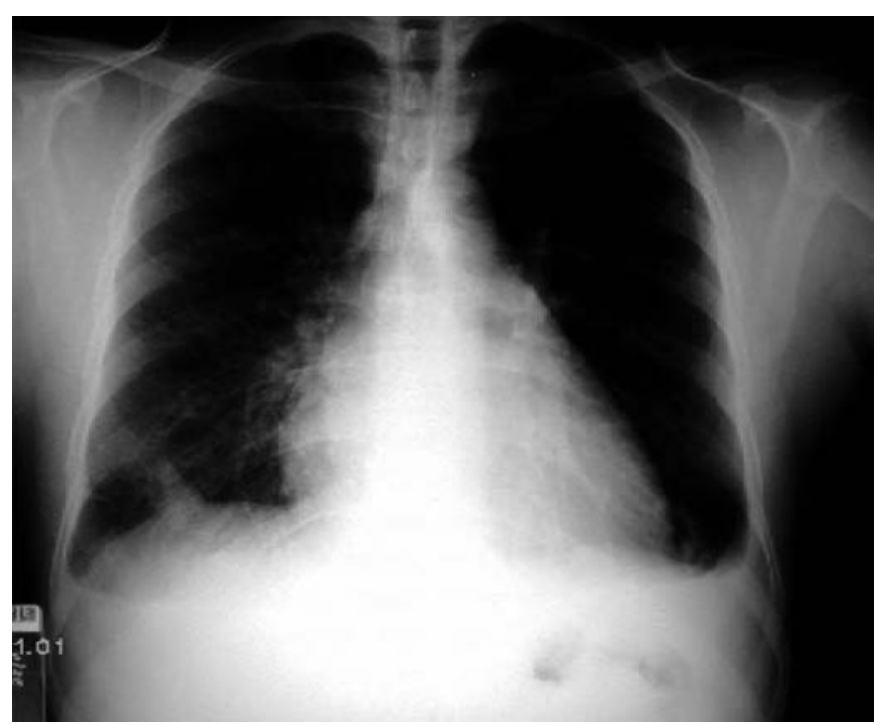

Fig. 1. Radiografía de tórax en la que se observa el derrame pleural. globulinas. Ferritina $8,2 \mathrm{n} / \mathrm{ml}$. TSH normal. Cobalamina y folatos nomrales. IgE normal. F reumatoide negativo. Proteína $\mathrm{C}$ reactiva normal. ANA negativo. ANCA negativo. Serología Lúes inmunoblot positivo. Serología de virus hepatitis B Ag HBs positivo, anti-Hbe positivo. PCR de virus B se detecta genoma. Serología virus $C$ negativa. Serología virus VIH negativa. Líquido cefalorraquídeo: recuento y formula normales; glucosa, proteínas y ADA normales; serología VDRL antc anti-treponema pallidum IgM + IgG negativos; $\mathrm{ZN}$ negativo. Test de Coombs directo negativo. Sedimento de orina normal, proteinuria de 24 horas negativa. Sangre en heces negativa. La radiografía de tórax era normal. En el electrocardiograma se apreciaba una HVI. El ecocardiograma reveló una hipertrofia concéntrica de ventrículo izquierdo ligera/moderada, regurgitación aórtica funcional ligera/mínima; dilatación ligera de AI; IT ligera con signos de HTSP ligera; derrame pericárdico ligero/mínimo; tamaño y contractilidad conservados. La prueba de esfuerzo fue negativa. Las ecografías abdominal y urológica no presentaron patología. La gastroscopia fue normal. Rechazó seguir el estudio de su patología.

La evolución fue buena se retiró el tratamiento con diclofenaco, se transfundieron 2 concentrados de hematíes, y se realizó tratamiento deplectivo con torasemida desapareciendo los edemas y el derrame pleural. El dolor de rodilla desapareció. La hipertensión arterial persistió después de la suspensión del fármaco.

Los AINEs se encuentran entre los grupos de fármacos más prescritos y consumidos en el mundo, llegando hasta el $10 \%$ del total de prescripciones(1). Presentan una baja frecuencia de efectos adversos, pero debido al gran número de pacientes que los consumen, hace que las complicaciones, principalmente renales y gastrointestinales, sean un problema sanitario importante (2).

El principal mecanismo de acción de los AINEs es la inhibición de la actividad de la enzima ciclooxigenasa, lo que lleva a una inibición de la síntesis de prostanoides (2-3).

Entre las Prostaglandinas (PG) más importantes del riñón están la PGE2 que se relaciona con la regulación de la reabsorción de sodio en la porción gruesa ascendente del asa de Henle y en el túbulo colector, y actúa como factor contrarregulador bajo condiciones de aumento de la reabsorción de sodio(4) ; la PGI2 o prostaciclina que aumenta la secreción de potasio al estimular la secreción de renina y activar el sistema renina-angiotensina-aldosterona. Menos importancia tiene la PGF2 $\alpha$, pero también aumenta la excreción de sodio y agua, y también presenta actividad vasoconstrictora; la PGD2 es vasodilatadora y el TXA2 es vasoconstrictor(5). Con la inhibición de la producción de PGE2 se produce un aumento de la reabsorción de sodio, que puede causar edema periférico, siendo este el efecto secundario más frecuente $(2,6)$. La retención de líquido aparece generalmente al principio del tratamiento, observándose en un 5\% de pacientes, llegando en algunos casos hasta el $25 \%$ de casos (1). Esta retención la mayor parte de las veces es leve, aumentando el paciente entre 1 ó $2 \mathrm{~kg}$ de peso, pero en otros casos la retención es mayor lo que lleva a un gran aumento de peso, grandes edemas e incluso cuadros de insuficiencia cardiaca. El paciente presentado no tenía criterios de insuficiencia cardíaca y presentaba una buena función ventricular.

La anemia se prodría atribuir a sangrado digestivo. El mayor porcentaje de efectos adversos de los AINEs tiene lugar a nivel gastrointestinal, En algunos estudios se ha observado que el tratamiento con AINEs a dosis medias o bajas, eleva el riesgo de complicaciones digestivas entre 2 y 3 veces. La localización más frecuente de las lesiones es la porción gastroduodenal, pero hay que tener en cuenta que las lesiones pueden aparecer desde el esófago hasta el recto. Las lesiones van desde el eritema hasta la ulceración, y se pueden observar en el 20-30 \% de pacientes que han seguido tratamiento con AINEs, que algunos casos cursan de forma asintomática (1).

Las PGE2 y la PGI2 tienen un efecto protector debido a que reducen la secreción ácida del estómago, producen dilatación de los vasos de la mucosa gástrica, aumentan la secreción de moco 
que forma una barrera física con función protectora y estimulan la secreción de bicarbonato. Por esto la inhibición de la producción de PGs E2 hace se pierda la protección natural del estómago (6).

La inhibición de las prostaglandina también se ha relacionado con la elevación de la presión arterial, debido a que la inhibición de la síntesis de PGs renales produce una retención de sodio y agua, y la falta de producción de PGI2 en algunos casos producirá un aumento, de las resistencias vasculares periféricas. En el caso expuesto el paciente probablemente era un hipertenso no diagnosticado ya que las cifras tensionales no se normalizaron tras la retirada del diclofenaco.

\section{F. J. Nicolás-Sánchez, R. M. Sarrat-Nuevo, L. Fernández- Cabrera, J. Cabau-Rubies}

\section{Servicio de Medicina Interna. Hospital de Santa María. Lleida}

1. Day MD, Morrison B, Luza A, Castaneda O, Strusberg A, Nahir M, et al. A randomised trial of the efficacy and tolerability of the COX-2 inhibitor rofecoxib vs ibuprofen in patients with osteoarthritis. Arch Intern Med 2000; 160: 1781-7.

2. Pepper G. Nonsteroidal anti-inflammatory drugs. New perspectives on a familiar drug class. Nursing Clinics North America 2000; 223-44.

3. Brooks P, Day R. Nonsteroidal anti-inflammatory drugs- Differences and similarities. N Eng J Med 1991; 324 (24): 1716-25.

4. Brater C. Effects of nonsteroidal anti-inflammatory drugs on renal function: focus on cyclooxygenase -2-selective inhibition. Am J Med 1999; 107 (6A): 65S-71S.

5. Whelton A, Hamilton C. Nonsteroidal anti-inflammatory drugs: Effects on kidney function. J Clin Pharmacol 1991; 31: 588-98.

6. Insel P. Analgesic-antipyretic and antiinflammatory agents and drugs employed in the treatment of gout. En: Hardman JG, Limbird LE, Molinoff PB, Ruddon RW, Goodman A, editors. Goodman \& Gillman. The pharmacological basis of therapeutics. 9th edition. McGraw-Hill 1996; 617-57.

\section{Utilidad de la serología rutinaria para el diagnóstico de la infección VIH}

\section{Sr. Director:}

Según la encuesta hospitalaria VIH/SIDA de 2006, el 63\% de los pacientes son diagnosticados por primera vez de esta infección en estadio de SIDA (1). Por otra parte se calcula que uno de cada cuatro infectados desconoce su enfermedad (2). Esto impide el acceso precoz a la terapia y favorece la transmisión inadvertida de la infección (3). Todo ello demuestra que las estrategias actualmente utilizadas para el diagnóstico son insuficientes.

A finales de 2006, los CDC propusieron que en la práctica clínica se realice la serología VIH de forma rutinaria a todos los pacientes entre 13 y 65 años $(2,4)$. Para evaluar la eficacia de esta recomendación debiéramos de conocer la frecuencia con la que las personas infectadas pero no diagnosticadas, demandan asistencia sanitaria, en otras palabras iacuden estos enfermos a los servicios de salud antes del diagnóstico?.

Para contestar esta pregunta y evaluar así el impacto de los criterios de los CDC, hemos revisado la información clínica de los pacientes diagnosticados por primera vez de esta infección en nuestra Area Sanitaria. Esta comprende una población de 220.000 personas, y los adultos con infección VIH reciben asis- tencia centralizada en la Unidad de Infectología del Hospital. Se seleccionaron los pacientes diagnosticados por primera vez de infección VIH desde agosto de 2002 hasta febrero de 2007, excluyéndose los casos de primoinfección y aquellos que procedieran de otras áreas. A continuación se revisaron sus Historias Clínicas y la base de datos de Análisis Clínicos del Area de Salud en vigor desde 1999.

Con estas características se encontraron 27 enfermos, 21 de ellos varones. La edad media era de $43 \pm 12$ años (27-77) y 2 superaban los 65 años. La vía de transmisión fue heterosexual en 18 casos (67\%), homosexual en 6 (22\%), y parenteral en $3(11 \%)$. En el momento del diagnóstico, 8 (30\%) se encontraban en estadio A, $4(15 \%)$ en estadio B y $15(55 \%)$ en estadio C. La media de CD4 fue de $219 \pm 293$ células $/ \mathrm{mm}^{3}$ (1-1100), y la mediana de 66 células $/ \mathrm{mm}^{3}$ (rango intercuartil, 370). Tras el diagnóstico, 5 fallecieron precozmente (en los tres primeros meses), 4 por causas directamente atribuibles a la infección VIH.

Se comprobó que a 19 pacientes $(70 \%)$ se les había realizado analítica previa al diagnóstico de la infección VIH, y a 15 en más de una ocasión. Las peticiones procedían de Atención Primaria en 14 casos, del Hospital en 16, y de ambos en 12. Dentro del Hospital, el mayor número de solicitudes analíticas se cursaron desde el Servicio de Urgencias.

Según los datos encontrados, si se hubiese realizado rutinariamente la serología VIH, estos 19 pacientes podrían haber conocido el diagnóstico entre 3 y 84 meses antes (media, 35 meses). No se han encontrado diferencias en la edad, estadio, vía de transmisión o recuento de CD4 entre el grupo con determinaciones analíticas previas y el resto.

Por lo tanto, aplicando la propuesta de los CDC (sin tener en cuenta la edad), el $70 \%$ de los pacientes hubiera conocido su enfermedad, como media, 35 meses antes. Es interesante señalar que esta estrategia hubiera beneficiado a todos los fallecidos precozmente. En la Bibliografía solo hemos encontrado un estudio similar, promovido por los CDC, y las cifras comunicadas son superponibles (5).

Estos hallazgos, aunque limitados, apoyan la utilidad de los criterios propuestos por los CDC. Sin embargo, en este estudio solo disponemos de datos de los enfermos diagnosticados de infección VIH, y esta información no puede extrapolarse al conjunto de infectados. No obstante, los resultados presentados, así como la experiencia de la práctica de la serología rutinaria en embarazadas, favorecen una postura más activa en el diagnóstico de esta infección. Quizás los criterios que actualmente seguimos para la realización de la serología VIH sean insuficientes e implican "oportunidades perdidas", tanto para el diagnóstico precoz como para el control de la epidemia (5).

\section{A. Chocarro Martínez, A. González López, I. García García, M. Aleixos Zuriaga}

Servicio de Medicina Interna. Hospital "Virgen de la Concha". Zamora

1. Encuesta Hospitalaria de Pacientes VIH/SIDA. Resultados 2006. Disponible en: http://www.isciii.es/htdocs/pdf/encuesta_hosp.pdf

2. Centers for Disease Control and Prevention. Revised recommendations for HIV testing of adults, adolescents, and pregnant women in healthcare settings. MMWR Recomm Rep 2006; 55 (RR-14): 1-17.

3. Janssen RS. HIV testing: Rationale for changing recommendations. Top HIV Med 2007; 15: 6-10.

4. Campos-Outcalt D. Time to revise your HIV testing routine. J Fam Pract 2007; 56: 283-4

5. Centers for Disease Control and Prevention. Missed opportunities for earlier diagnosis of HIV infection-South Carolina, 1997-2005. MMWR Morb Mortal Wkly Rep 2006; 55: 1269-72. 